\title{
The Robustness of an Efficient Probabilistic Data-Based Tool for Simulating the Nonstationary Response of Nonlinear Systems
}

\author{
Andrew W. Smyth (Corresponding Author) \\ Dept. of Civil Engineering \& Engineering Mechanics \\ Columbia University, New York, New York, USA \\ smyth@civil.columbia.edu \\ Sami F. Masri \\ School of Engineering \\ University of Southern California, Los Angeles, California, USA \\ masri@usc.edu
}

\begin{abstract}
The stochastic response of dynamic systems has been an area of considerable interest for some time in the analysis of risk and structural reliability. The authors, in previous work, have developed a method which can analyze the response of linear multi-degree-of-freedom systems to completely general data-based nonstationary excitations in a highly efficient and analytical form. The authors extended this work to nonlinear system response by using equivalent linearization techniques. This paper explores the range of application of the extension to the analysis of nonlinear systems through the use of real and simulated data-sets. In particular, sensitivity issues of non-Gaussianity of the excitation data, and the degree of response nonlinearity of the dynamic system, are investigated for their effect on the estimated response using this equivalent linearization based approach.
\end{abstract}

Keywords: Equivalent Linearization, Random Vibrations, Nonstationary, Karhunen-Loeve

\section{Background}

In Smyth and Masri [1], the authors developed a new highly computationally efficient method for simulating the response of nonlinear dynamic systems subject to nonstationary excitation processes. The semi-analytical method, which only involves numerical integration in the final simulation step, is built on the data condensation of measured excitation process data through the Karhunen-Loeve expansion, and through a special polynomial approximation of the eigenvectors 
of the covariance matrix of the process. The fundamental starting point for the development of the new technique is in the compact analytical data approximation used for the nonstationary excitation. After applying Karhunen-Loeve spectral decomposition to the measured excitation process covariance matrix $\mathcal{C}_{k}\left(t_{1}, t_{2}\right)$, and least-squares fitting the eigenvectors with orthogonal polynomials, the excitation data is condensed into the following approximate form:

$$
\hat{\mathcal{C}}_{k}\left(t_{1}, t_{2}\right)=\sum_{i=1}^{k} \lambda_{i} \sum_{j=0}^{m_{i}-1} \sum_{\ell=0}^{m_{i}-1} H_{i j} H_{i \ell} T_{j}\left(t_{1}^{\prime}\right) T_{\ell}\left(t_{2}^{\prime}\right),
$$

where the $\lambda_{i}$ 's are the truncated series of eigenvalues, the $T_{j}\left(t_{1}^{\prime}\right)$ 's are Chebyshev polynomials, and the $H$ 's are weighting coefficients for the Chebyshev polynomials in the least-squares fitting of the eigenvectors. In this equation, $t_{i}^{\prime}=2 t_{i} / t_{\max }-1$. For additional details the reader is referred to Masri et al. [2]. In effect, the conversion from measured data to analytical representation involves the approximation of the discrete eigenvectors of the excitation covariance matrix by analytical eigenfunctions (given as a polynomial series).

The pre-cursor to the solution of the nonlinear multi-degree-of-freedom system simulation problem, was the solution for general (i.e., without assuming proportional damping) linear systems of the form $\dot{\mathbf{x}}=\mathbf{A x}+\mathbf{B u}$, and was developed by the authors in a purely analytical, closed-form solution in 1998 (Smyth [3], Masri et al. [2]). The critical link from the linear to the nonlinear solution methodology was made through the use of equivalent linearization techniques. The formulation is briefly reviewed in the next section.

\section{Statistical Linearization and Nonstationary Excitation - A Review}

Equivalent linearization (see for example, Roberts and Spanos [4]) is the approximation of nonlinearities through approximate linear systems, so that one can use linear system solution methodologies to make nonlinear system analysis more tractable. Of course, equivalent linearization, comes with limitations, and those are that typically the method works well for weak nonlinearities, and often requires Gaussianity (across the ensemble) of the response process (see e.g., Lutes [5], and 
Smyth and Masri [1]). The vast majority of the equivalent linearization literature concerns stationary input and output processes. Here, the nonstationary excitation and response problem is briefly reviewed.

Consider the following nonlinear $n$ degree of freedom equation of motion

$$
\mathbf{M} \ddot{\mathbf{q}}(t)+\mathbf{C} \dot{\mathbf{q}}(t)+\mathbf{K q}(t)+\mathbf{g}(\mathbf{q}(t), \dot{\mathbf{q}}(t))=\mathbf{F}(t)
$$

where $\mathbf{g}$ is a nonlinearity dependent upon the system displacements and velocities, and $\mathbf{F}(t)$ is a random vector process of length $n$. After some manipulation, this nonlinear system can be written in its equivalent linear state-space form (see Roberts and Spanos [4]):

$$
\dot{\mathbf{z}}=\mathbf{G}(t) \mathbf{z}+\mathbf{f}
$$

where

$$
\begin{aligned}
& \mathbf{z}(t)=\left[\begin{array}{c}
\mathbf{q}_{0}(t) \\
\dot{\mathbf{q}}_{\mathbf{0}}(t)
\end{array}\right] \text { and } \mathbf{q}_{0}(t)=\mathbf{q}(t)-\boldsymbol{\mu}_{\mathbf{q}}(t) \\
& \mathbf{G}(t)=\left[\begin{array}{cc}
\mathbf{0} & \mathbf{I} \\
-\mathbf{M}^{-1}\left(\mathbf{K}+\mathbf{K}_{\mathbf{e}}\right) & -\mathbf{M}^{-1}\left(\mathbf{C}+\mathbf{C}_{\mathbf{e}}\right)
\end{array}\right] \\
& \mathbf{f}=\left[\begin{array}{c}
\mathbf{0} \\
-\mathbf{M}^{-1} \mathbf{F}_{\mathbf{0}}
\end{array}\right] \quad \text { and } \quad \mathbf{F}_{0}(t)=\mathbf{F}(t)-\boldsymbol{\mu}_{\mathbf{F}}(t)
\end{aligned}
$$

and where $\mathbf{C}_{\mathbf{e}}$ and $\mathbf{K}_{\mathbf{e}}$ are time-dependent equivalent linear terms which account for the nonlinear g; this contrasts with stationary statistical linearization problems where these matrices are constant.

The goal is to get a second order probabilistic description of the response state $\mathbf{z}$, i.e. $\mathbf{V}(t) \equiv$ $E\left\{\mathbf{z}(t) \mathbf{z}^{T}(t)\right\}$. The differential equation which defines the evolution of $\mathbf{V}(t)$ is

$$
\begin{gathered}
\dot{\mathbf{V}}=\mathbf{G}(t) \mathbf{V}+\mathbf{V G}^{T}(t)+\mathbf{U}(t)+\mathbf{U}^{T}(t) \\
\text { where } \mathbf{U}(t)=E\left\{\mathbf{z f}^{T}\right\}
\end{gathered}
$$

After incorporating the compact form of the excitation process, one may write $\mathbf{U}(t)$ as

$$
\mathbf{U}(t)=\sum_{i=1}^{k} \lambda_{i} \sum_{j=0}^{m_{i}-1} \sum_{\ell=0}^{m_{i}-1} H_{i j} H_{i \ell} T_{j}\left(t^{\prime}\right) \mathbf{U}^{(\ell)}(t)
$$

and after some further manipulation $\mathbf{U}^{(\ell)}(t)$ can be expressed as

$$
\dot{\mathbf{U}}^{(\ell)}(t)=\mathbf{G}(t) \mathbf{U}^{(\ell)}(t)+\mathbf{R} T_{\ell}\left(t^{\prime}\right)
$$


where $\mathbf{R}$ is a very simple constant matrix which defines the degrees of freedom to which the force is applied. For example, in the single input case

$$
\mathbf{W}_{\mathbf{f}}\left(t_{1}, t_{2}\right) \equiv E\left\{\mathbf{f}\left(t_{1}\right) \mathbf{f}^{T}\left(t_{2}\right)\right\}=\mathbf{R} \mathcal{C}_{s s}\left(t_{1}, t_{2}\right)
$$

This new solution is highly efficient, contrasting with an analogous decomposition-based solution by Roberts and Spanos [4] which is limited to a restrictive class of nonstationary excitations, and involves 3-index terms $\mathbf{U}^{(i j k)}(t)$ which increases the number of terms in the nested summations in Eq. (6). For a detailed comparison of the two approaches, the reader is referred to Smyth and Masri [1].

In Smyth and Masri [1], for general nonstationary excitations, the method was shown to perform extremely well relative to much more computationally intensive "exact" simulations performed through Monte Carlo simulations. Performance appeared to deteriorate somewhat for highly nonlinear system response coupled with a highly non-Gaussian real-world input process. Despite the slight performance degradation, the overall peak magnitude of the estimated RMS (root-mean-square) response was very close to the "exact" peak RMS response.

\section{Scope}

In this paper, the contrast between performance with a relatively low degree of non-Gaussianity versus a high level of non-Gaussianity in the excitation and response processes will be explored. In the previous presentation of the method a hardening Duffing oscillator was used. In this paper, a softening Duffing oscillator is used because of the additional inherent challenges. This nondimensional oscillator equation is given as

$$
\ddot{q}+2 \zeta \dot{q}+q+\lambda q^{3}=s(t)
$$

Throughout this paper $\zeta=0.1$ and $\lambda=-0.1$. Because $\lambda$ is negative this is a softening Duffing oscillator, and because it is the opposite sign of the stiffness (which is here 1), this leads to nonlinear behaviors which can be quite different from those of the hardening oscillator (as will be seen 
in a later displacement versus restoring force figure), and which present stability challenges for numerical solvers.

\section{Simulation Results}

In Smyth and Masri [1], it was shown that the methodology was highly accurate for estimating the response of nonlinear systems to synthetically generated Gaussian random nonstationary excitations. Therefore, here, one will begin by considering a more challenging situation, which is that of a measured non-Gaussian process. A set of almost 1200 earthquake ground motion acceleration records were collected from the 1999 Chi-Chi earthquake in Taiwan. These will be used as the basic excitation data source. Because they are of widely varying magnitudes etc., in order to make the ensemble more Gaussian (across the ensemble), the records were all normalized to have the same peak magnitude. In this study, the accelerations were actually assumed to be the excitation force $s(t)$. In addition, the time-scale was stretched to permit qualitative comparison with the results in

Smyth and Masri [1]. A sample of four realizations of the un-normalized excitation processes is shown in Figure 1. After normalization the non-Gaussian excitation process can be seen to become relatively more Gaussian. This can be seen from a comparison of the curtosis and skewness of the ensemble before and after the normalization step. In theory, for a perfectly Gaussian process one should have curtosis equal to 3 and skewness equal to zero. Figure 2 shows a comparison of the time-varying skewness and curtosis with and without normalization. Notice that the scales are not the same. Clearly the magnitudes of curtosis and skewness tend to be much larger in the case of no normalization.

\subsection{Response to a Somewhat Non-Gaussian Measured Excitation Process}

The softening Duffing oscillator was subjected to the normalized data ensemble by both direct Monte-Carlo simulation and using the equivalent linearization based estimation approach. The direct approach took several hours of computing time, and the equivalent linearization approach took about 30 seconds using the same integration algorithm with the same level of error tolerance. 
A sample phase-plane plot of the displacement vs. restoring force is shown in Figure 3. The restoring force is simply $r(t)=2 \zeta \dot{q}+q+\lambda q^{3}=s(t)-\ddot{q}$

The equivalent linearization approach is capable of providing the second-order output statistics of the response displacement and velocity, and these are compared with those calculated by the "exact" Monte-Carlo approach in Figure 4. Clearly the accuracy of the method is excellent in this case despite the reasonable degree of non-Gaussianity of the normalized excitation process.

\subsection{Response to a Highly Non-Gaussian Measured Excitation Process}

The same softening Duffing oscillator was subjected to the un-normalized data ensemble by direct Monte-Carlo simulation and using the equivalent linearization based approach. Again, the direct approach took several hours of computing time, and the equivalent linearization approach took about 30 seconds. A sample phase-plane plot of the displacement vs. restoring force is shown in Figure 5. Clearly for this realization the nonlinear response behavior is quite nonlinear. In this case the transient response estimate shown in Figure 6 was not as accurate as before.

\subsection{Response to Synthetic Excitation of Varying Non-Gaussianity}

Although this method was principally designed to efficiently handle data-based excitation processes, it is useful to consider synthetically generated excitation process data through a set of controlled input parameter simulations to determine the practical limits of the accuracy of this approximate method. It is assumed here that the excitation processes will have zero mean and symmetric probability distributions. In particular, from experience gained in [1], the probability densities across the ensemble at a given time for many earthquake data ensembles resembles a truncated Cauchy distribution. The probability density function of a Cauchy distribution is given by:

$$
p(x)=\frac{1}{\pi} \frac{\frac{1}{2} \Gamma}{(x-\mu)^{2}+\left(\frac{1}{2} \Gamma\right)^{2}}
$$

where $\Gamma$ is the full width at half maximum and $\mu$ is the mean, which is here assumed to be zero. A comparison of Cauchy probability density functions with various $\Gamma$ values is shown in Figure 
7 together with a Gaussian distribution of unit variance. Note the vertical axis is provided on a logarithmic scale to highlight the difference in the tails of the distributions.

Wide-band random data realizations with a normal distribution were low-pass filtered (in order to sufficiently excite the nonlinear range of the oscillator) and then, through a mapping were converted to have Cauchy probability densities across the ensemble for all $t_{k}$. Note that through this direct mapping the correlation structure of the original data was not preserved. In order to synthesize the amplitude modulation, the envelope function presented in Jennings et al. [6] was used, and which builds as $\left(t / t_{1}\right)^{2}$ up to $t=t_{1}$, then remains constant at unity until $t=t_{2}$, and then decays as $\exp \left[-c\left(t-t_{2}\right)\right]$.

As mentioned, an attempt was made to scale the input excitation in order to produce a similar level of nonlinear response for each case. The $\Gamma$ parameter was varied from 0.1 to 3 to explore the robustness of the methodology. A sample of the input realizations is shown for various $\Gamma$ values is shown in Figure 8. A representative RMS displacement and velocity comparison of the exact and estimated transient response is shown in Figure 9. Clearly the accuracy is quite good. Acknowledging that it is extremely difficult to make direct quantitative comparisons of the performance for different input distributions one does notice, that for this set of cases, the norm error in the RMS displacement reponses was less than $1 \%$. Based on the previous real-world un-normalized data example we know that this kind of performance is by no means assured. It may be that highly heterogenous magnitude excitation data which may look like a Cauchy distribution across the ensemble produces some very large responses, and some relatively small ones. The response statistics are correspondingly phenomenologically different than a normalized data-set (as considered here in this set of synthetic experiments) which also satisfies a given Cauchy distribution across the ensemble.

In addition to the Cauchy distribution, a Beta distribution was also considered to explore the impact of using another non-Gaussian distribution. The Beta distribution used for the mapping was limited from -5 to 5 as shown in Figure 10. Interestingly again, agreement between estimated and "exact" response statistics was excellent with norm error of just under $1 \%$ for all three cases 
of $r=q=2,4$, and 10 in the Beta distribution.

\section{DISCUSSION}

It is clear from this simulation-based comparison, that deterioration in the accuracy of the approximation approach is dependent on the increased non-Gaussianity in the excitation process particularly if produced by a large range of amplitudes of the realizations within an ensemble. It should be noted, that strictly-speaking, for the way this particular equivalent linearization scheme was implemented (Smyth and Masri [1]), that actually the Gaussianity condition is on the response, which, in this case, is the cubic term associated with the displacement, and is approximated with a time-varying linear term. (Time-varying, because this is a nonstationary simulation.) Therefore, it actually makes more sense to compare the curtosis and skewness of the response to determine their relative Gaussianity. This comparison is shown in Figure 12 for the normalized and un-normalized earthquake data example.

Because a softening Duffing oscillator was considered here, it was difficult to generate highly nonlinear responses, beyond the kind observed in Figure 5. This difficulty was, for the reason mentioned earlier, that beyond a certain displacement the negative cubic term outweighs the linear stiffness and in effect produces negative stiffness. This means that the entire set of simulations here resides within one of the constraints of equivalent linearization: and that is the constraint of weak nonlinearity. Deterioration in performance for a hardening oscillator with $\lambda=+0.3$, and responding highly nonlinearly was observed. Naturally the maximum error occurs during the largest response, i.e. when the system behaves most nonlinearly. The same set of synthetic experiments was performed with the various Cauchy distributions, and the norm error in displacement was between $5-10 \%$ and the norm error in velocity was typically around $2 \%$. A representative phase plane and displacement response comparison is shown in Figure 11. Again, the error seemed to be more dependent on the magnitude of the response (i.e. the amount of nonlinearity) rather than the input distribution. 
Overall, despite the increased non-Gaussianity of the excitation process, the estimated response is still quite accurate, and does again yield a reasonable estimate of the peak response statistics. It has been noted in Lutes [5] and elsewhere, and is confirmed here for nonstationary processes, that in practice, relatively good approximation accuracy of response variances $E\left\{q(t)^{2}\right\}$ by statistical linearization approaches is observed even with relatively non-Gaussian response input and output processes. Although not explicitly derived in Smyth and Masri [1], the formulation can be extended to calculating the off-diagonal terms in the covariance matrix of the response, i.e., $E\left\{q\left(t_{j}\right) q\left(t_{k}\right)\right\}$

where $t_{j} \neq t_{k}$. It would be interesting to see if the relative robustness to non-Gaussianity is still observed for the accuracy of the off-diagonal terms of the response covariance matrix.

While the methodology is already formulated for multi-degree-of freedom systems with multiple inputs (Smyth and Masri [1]), future simulation work will be required to explore its robustness for this more challenging problem. In addition, hysteretic nonlinearities require an extra set of requirements on the input and response processes for equivalent linearization. For example, Caughey [7] required the assumption of narrow-bandedness for the linearization of bilinear hysteresis. These issues remain to be explored for this general nonstationary response analysis technique.

\section{Acknowledgments}

This study was supported in part by the National Science Foundation and the Air Force Office of Scientific Research. 


\section{References}

[1] A. W. Smyth and S. F. Masri. Nonstationary response of nonlinear systems using equivalent linearization with a compact analytical form of the excitation process. Probabilistic Engineering Mechanics, 17(1):97-108, January 2002.

[2] S. F. Masri, A. W. Smyth, and M. I. Traina. Probabilistic representation and transmission of nonstationary processes in multi-degree-of-freedom systems. Jo. of Applied Mechanics, ASME, 65:398-409, June 1998.

[3] A. W. Smyth. Experimental and Analytical Studies in Nonlinear System Identification and Modeling for Structural Control. Ph.d. thesis, University of Southern California, Los Angeles, CA, May 1998.

[4] J. B. Roberts and P. D. Spanos. Random Vibration and Statistical Linearization. John Wiley \& Sons, New York, NY, 1990.

[5] L. D. Lutes. The gaussian assumption in equivalent linearization. In Eigth ASCE Speciality Conference on Probabilistic Mechanics and Structural Reliability, PMC2000, number PMC2000-334. Univ. of Notre Dame, August 2000.

[6] P.C. Jennings, G.W. Housner, and N.C. Tsai. Simulated earthquake motions. Technical report, Earthquake Engineering Research Laboratory, California Inst. of Technology, April 1968.

[7] T.K. Caughey. Random excitation of a system with bilinear hysteresis. ASME Jo. of Applied Mechanics, 27:649-652, 1960. 


\section{List of Figures}

1 Four sample realizations from the measured excitation process ensemble of 1191

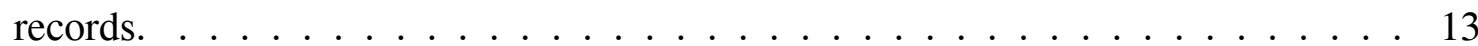

2 Comparison of the skewness and curtosis of the excitation process ensemble with and without normalization. It should be noted that after normalization the data was multipled by a scale factor of 0.65 , and the un-normalized ensemble was multiplied by a scale factor of 1.5 . This scaling was done in order to exercise the peak nonlinear response to a similar degree. . . . . . . . . . . . . . . . . . . . 14

3 Displacement versus restoring force sample response from the normalized excita-

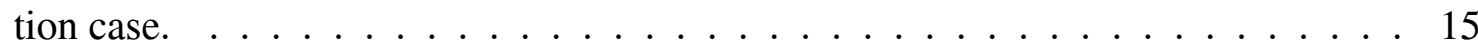

4 A comparison of the exact and estimated root-mean-square of the transient displacement and velocity responses. . . . . . . . . . . . . . . 16

5 Displacement versus restoring force sample response from the un-normalized earthquake data excitation case. Although this particular response realization was quite nonlinear this should not be considered to be representative of all of the responses.

6 A comparison of the exact and estimated mean-square (variance) of the transient displacement and velocity responses due to the un-normalized earthquake data ex-

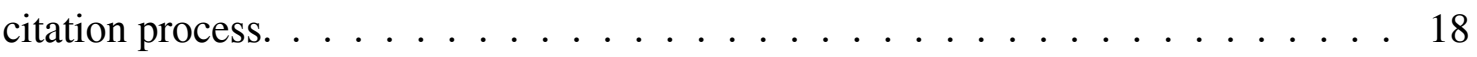

7 A comparison of the normal distribution used to generate the original data realizations together with the Cauchy distributions to which the data is mapped. This is before the entire synthetic data set was scaled to exercise the nonlinear element to a similar degree. ......................... 19

8 A sampling of the synthetic excitation realizations for different $\Gamma$ values in the Cauchy distribution. . . . . . . . . . . . . . . . 20 
9 A comparison of the exact and estimated root-mean-square (variance) of the transient displacement and velocity responses due to the normalized synthetic excitation process which adheres to a Cauchy distribution across the ensemble with

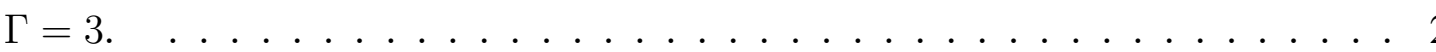

10 A comparison of the normal distribution used to generate the original data realizations together with the Beta distributions to which the data is mapped. This is before the entire synthetic data set was scaled to exercise the nonlinear element to

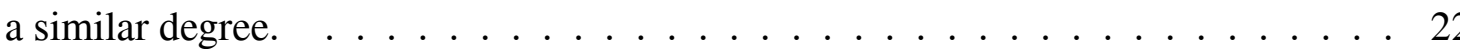

11 A representative phase plane response of the hardening oscillator subjected to an ensemble with Cauchy distribution and $\Gamma=0.6$. Also shown is a comparison of the transient rms displacement response. . . . . . . . . . . . . . . . 23

12 A comparison of the skewness and curtosis of the response displacement due to the normalized and un-normalized non-Gaussian excitation processes. Notice that the magnitude in the un-normalized case (right column) is much larger than that for the normalized case. . . . . . . . . . . . . . . . . 24 

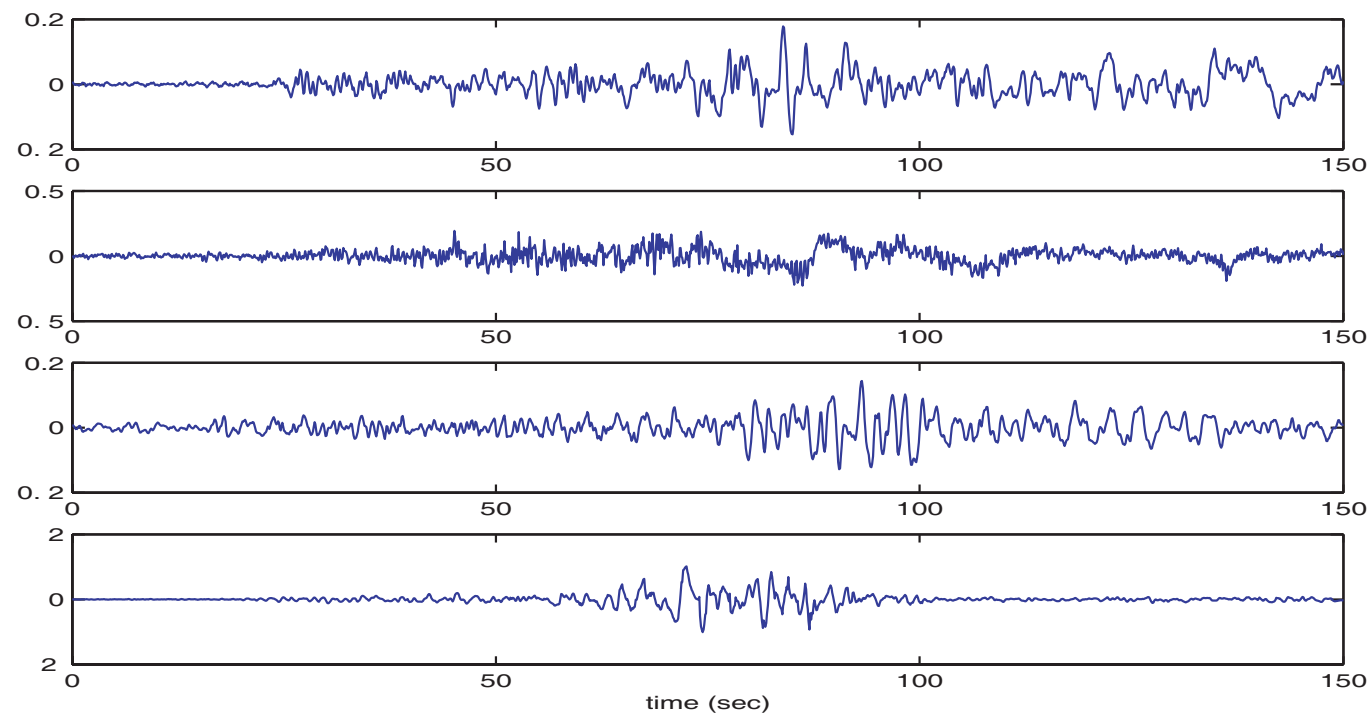

Figure 1: Four sample realizations from the measured excitation process ensemble of 1191 records. 

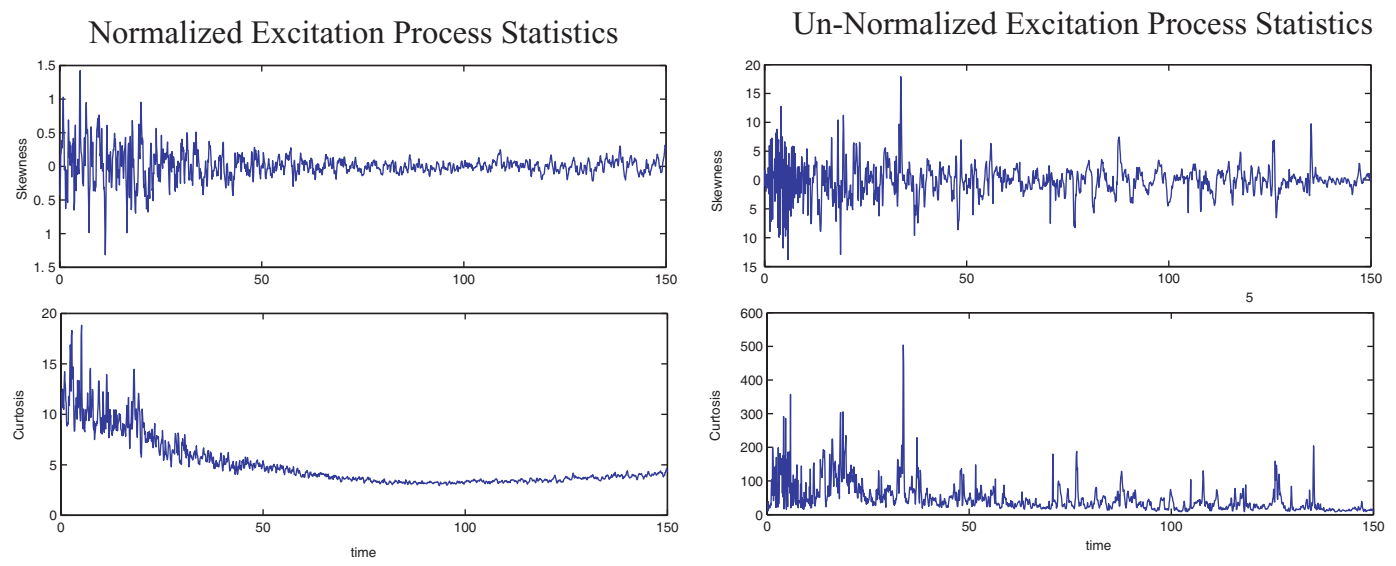

Figure 2: Comparison of the skewness and curtosis of the excitation process ensemble with and without normalization. It should be noted that after normalization the data was multipled by a scale factor of 0.65 , and the un-normalized ensemble was multiplied by a scale factor of 1.5 . This scaling was done in order to exercise the peak nonlinear response to a similar degree. 


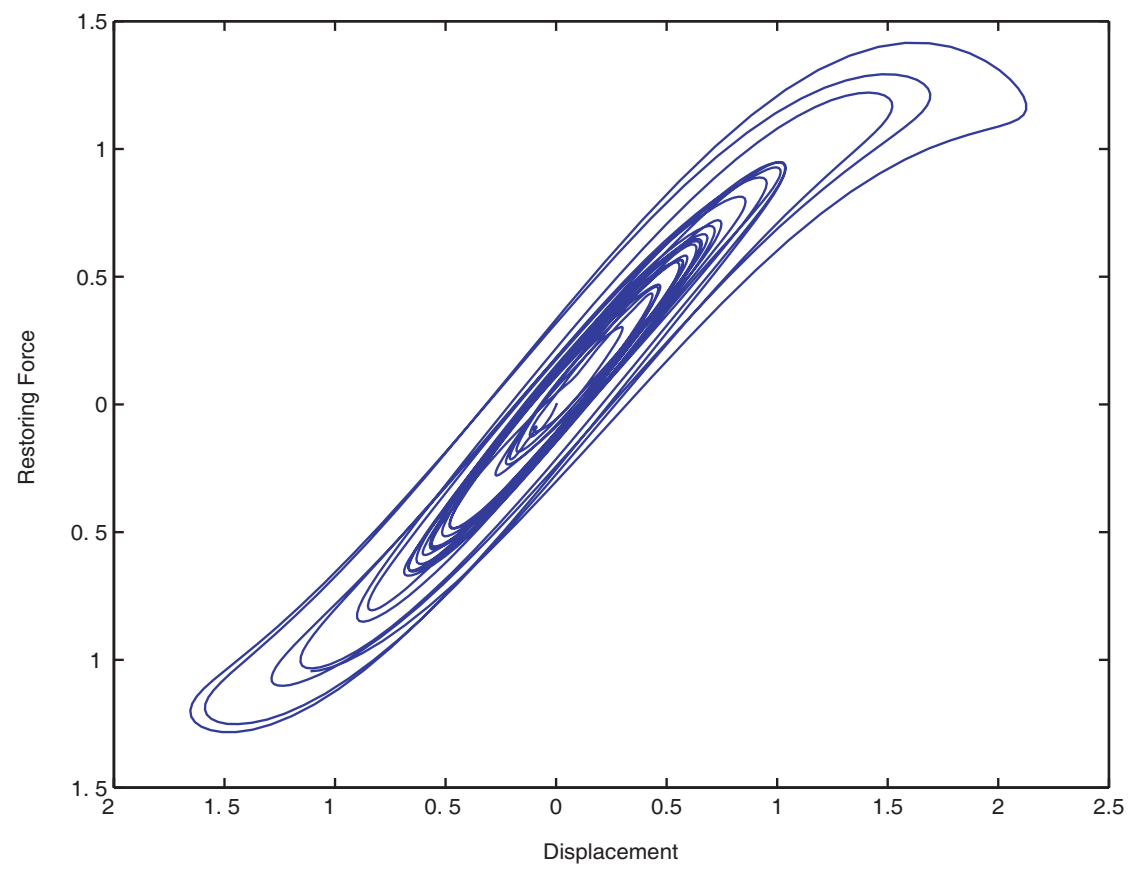

Figure 3: Displacement versus restoring force sample response from the normalized excitation case. 

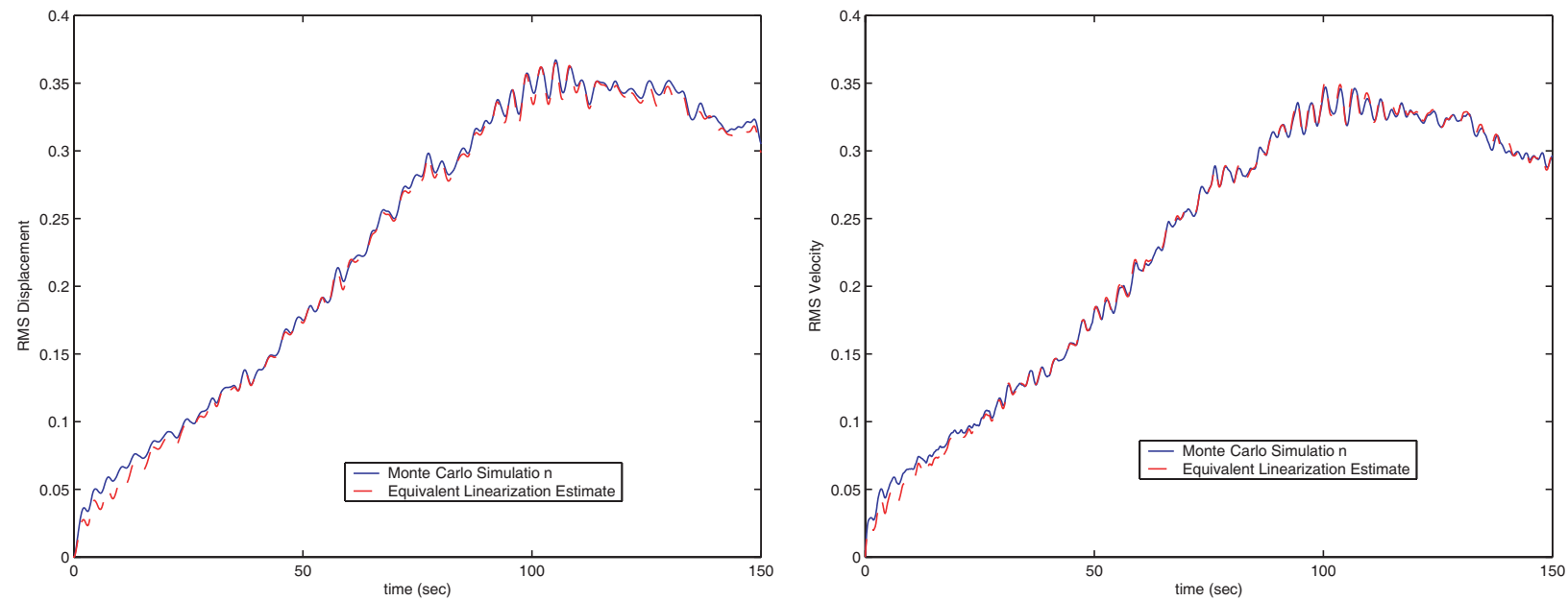

Figure 4: A comparison of the exact and estimated root-mean-square of the transient displacement and velocity responses. 


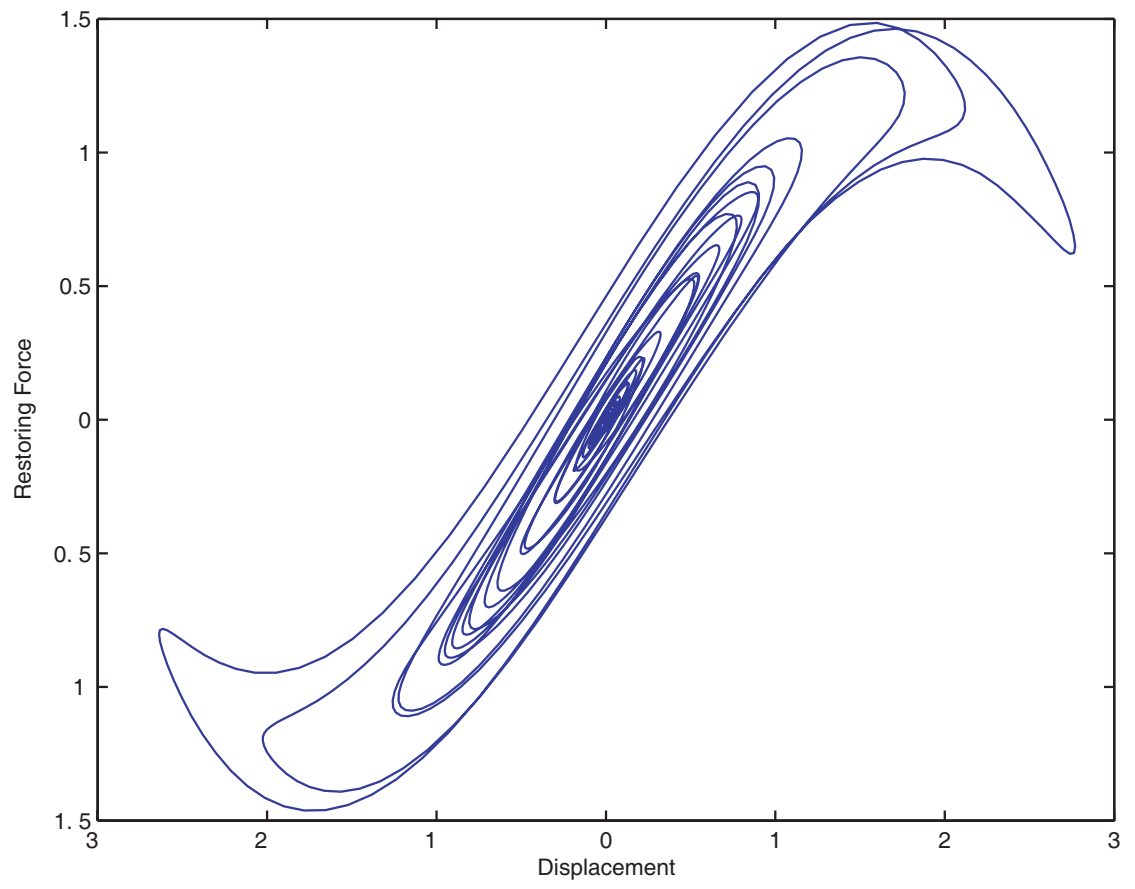

Figure 5: Displacement versus restoring force sample response from the un-normalized earthquake data excitation case. Although this particular response realization was quite nonlinear this should not be considered to be representative of all of the responses. 

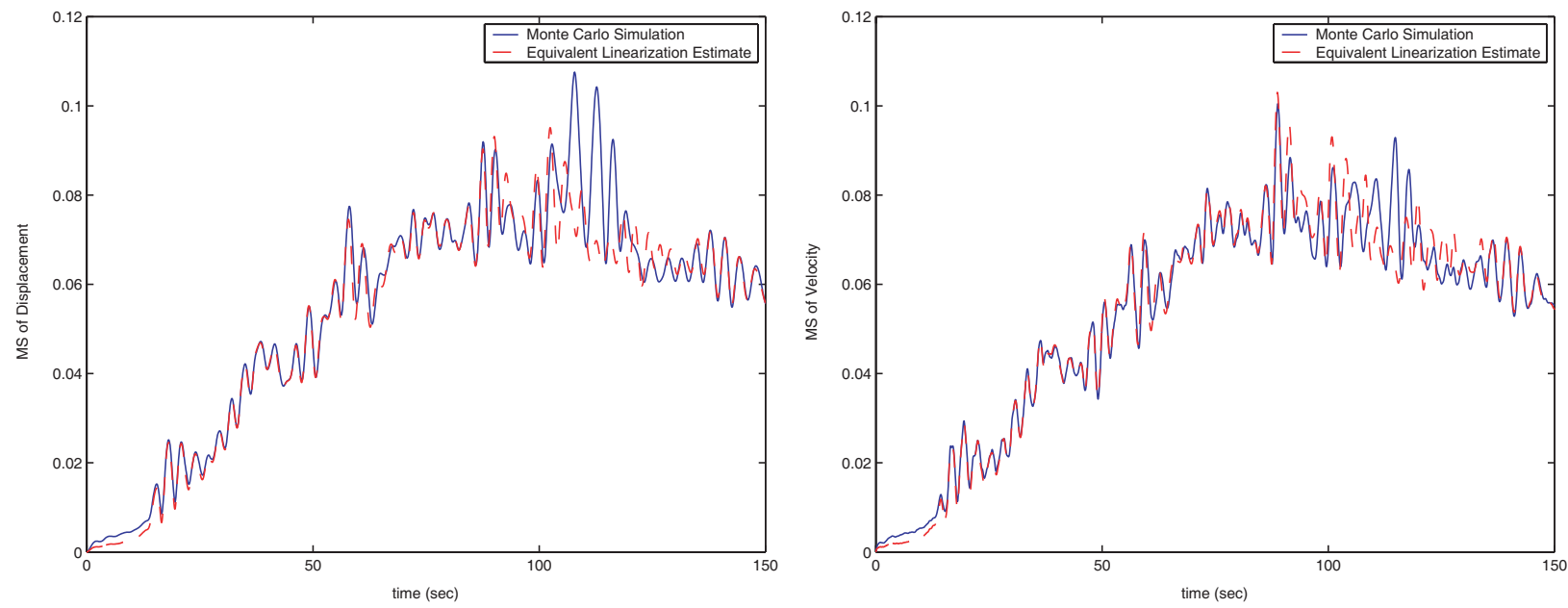

Figure 6: A comparison of the exact and estimated mean-square (variance) of the transient displacement and velocity responses due to the un-normalized earthquake data excitation process. 


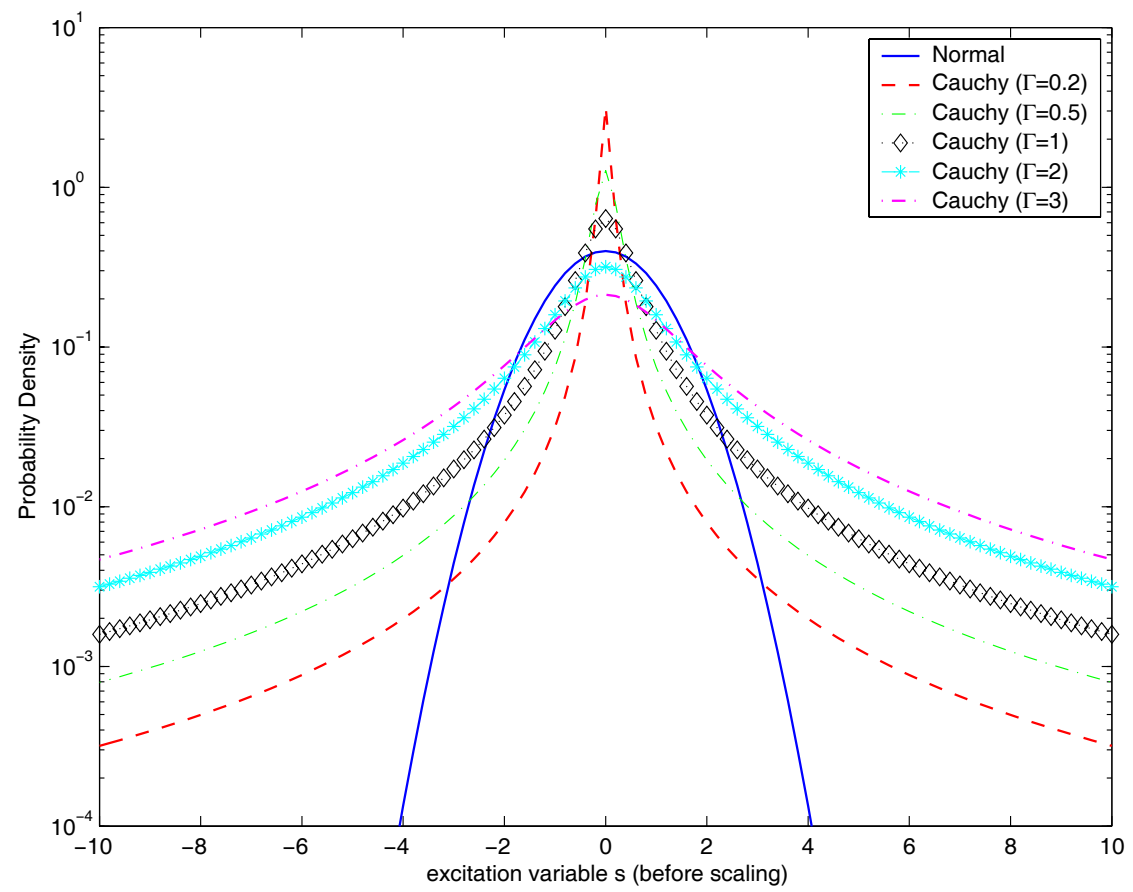

Figure 7: A comparison of the normal distribution used to generate the original data realizations together with the Cauchy distributions to which the data is mapped. This is before the entire synthetic data set was scaled to exercise the nonlinear element to a similar degree. 

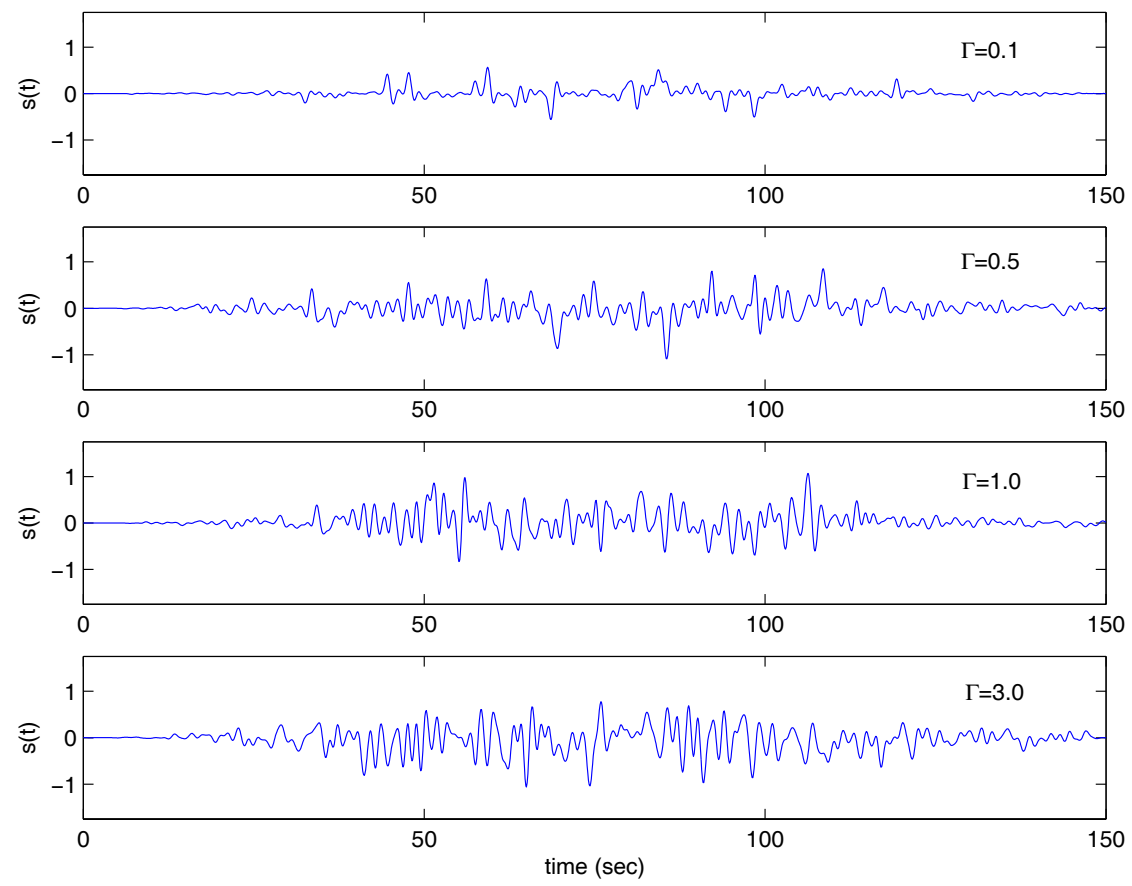

Figure 8: A sampling of the synthetic excitation realizations for different $\Gamma$ values in the Cauchy distribution. 

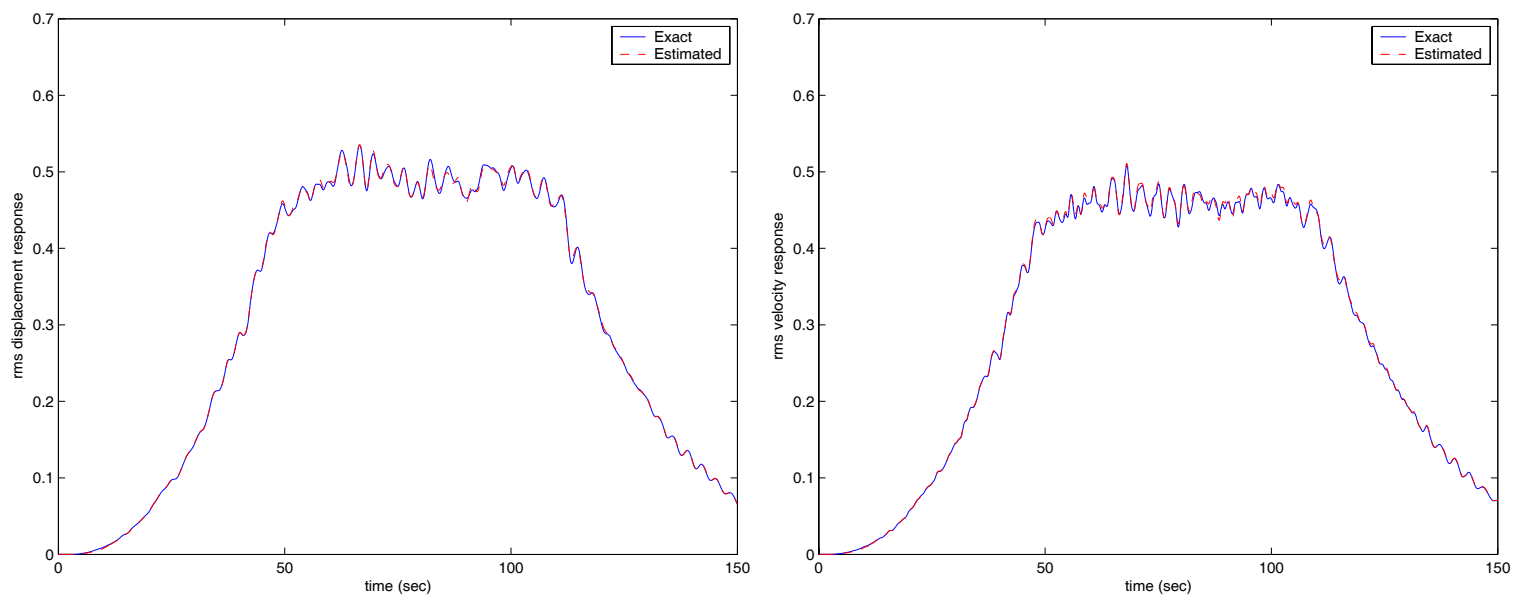

Figure 9: A comparison of the exact and estimated root-mean-square (variance) of the transient displacement and velocity responses due to the normalized synthetic excitation process which adheres to a Cauchy distribution across the ensemble with $\Gamma=3$. 


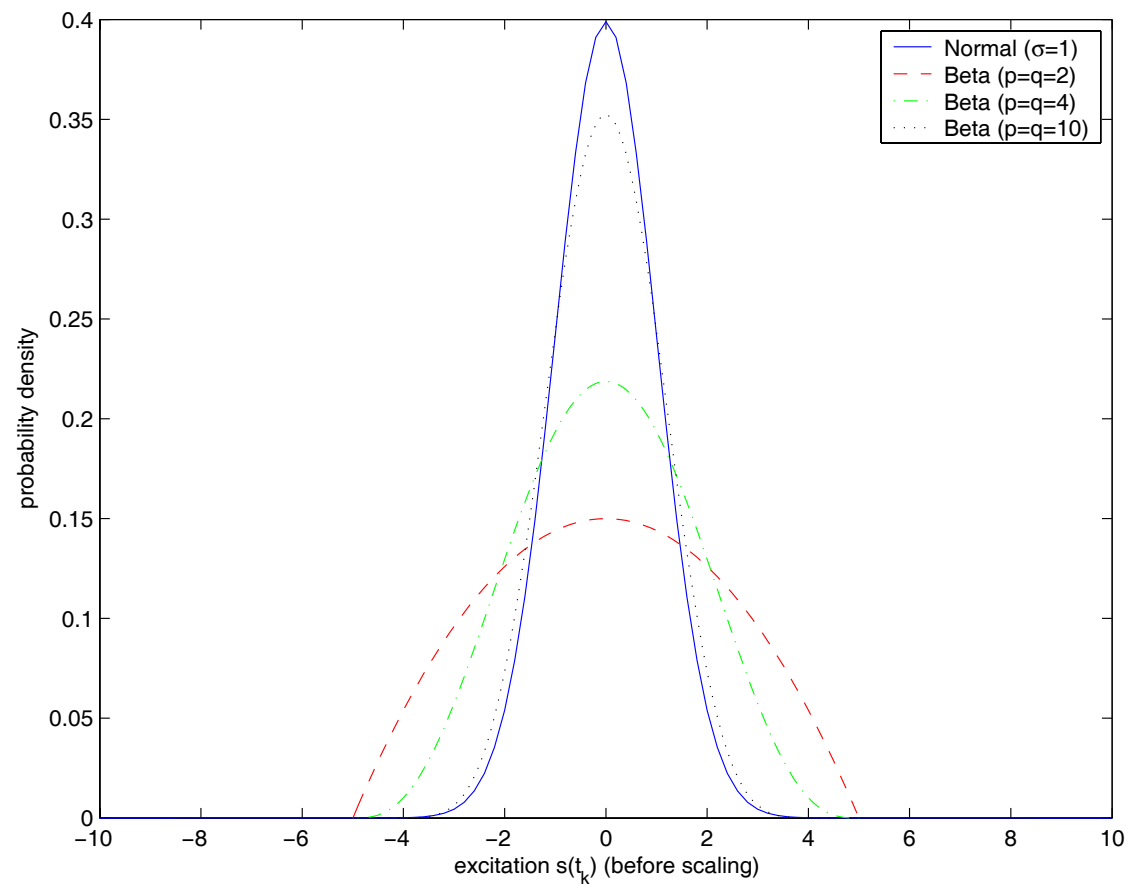

Figure 10: A comparison of the normal distribution used to generate the original data realizations together with the Beta distributions to which the data is mapped. This is before the entire synthetic data set was scaled to exercise the nonlinear element to a similar degree. 

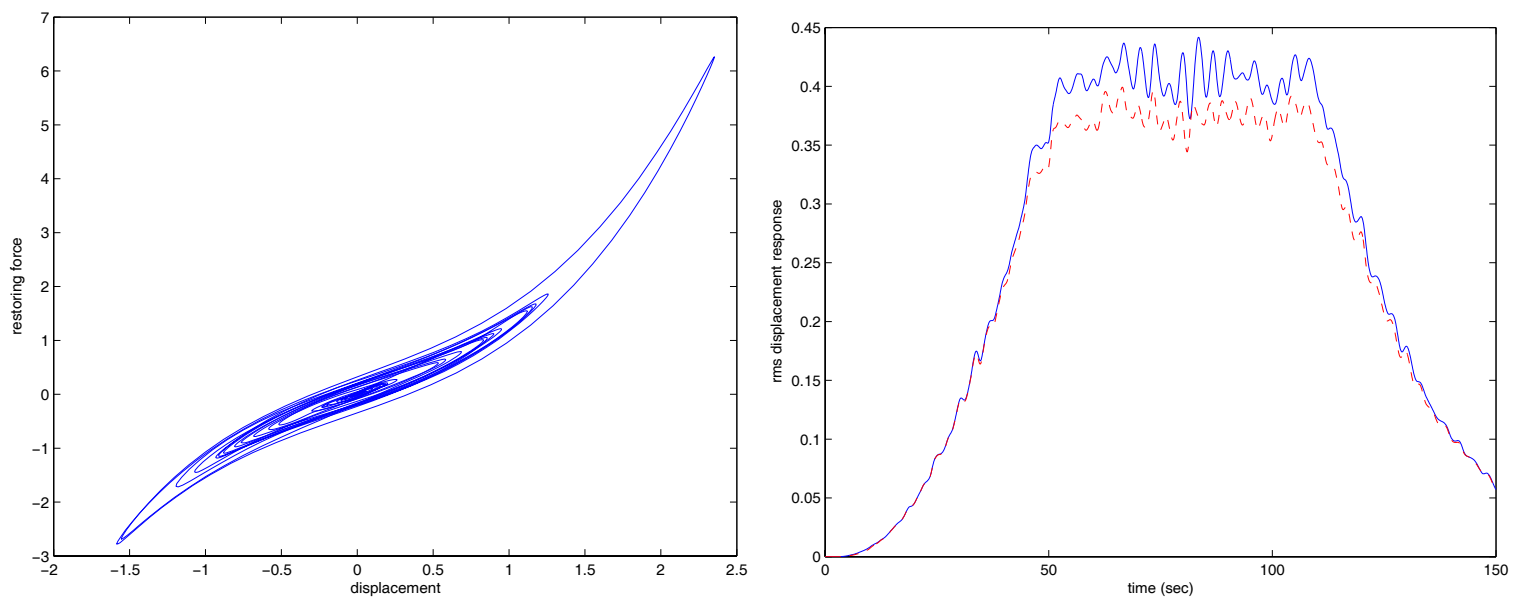

Figure 11: A representative phase plane response of the hardening oscillator subjected to an ensemble with Cauchy distribution and $\Gamma=0.6$. Also shown is a comparison of the transient rms displacement response. 

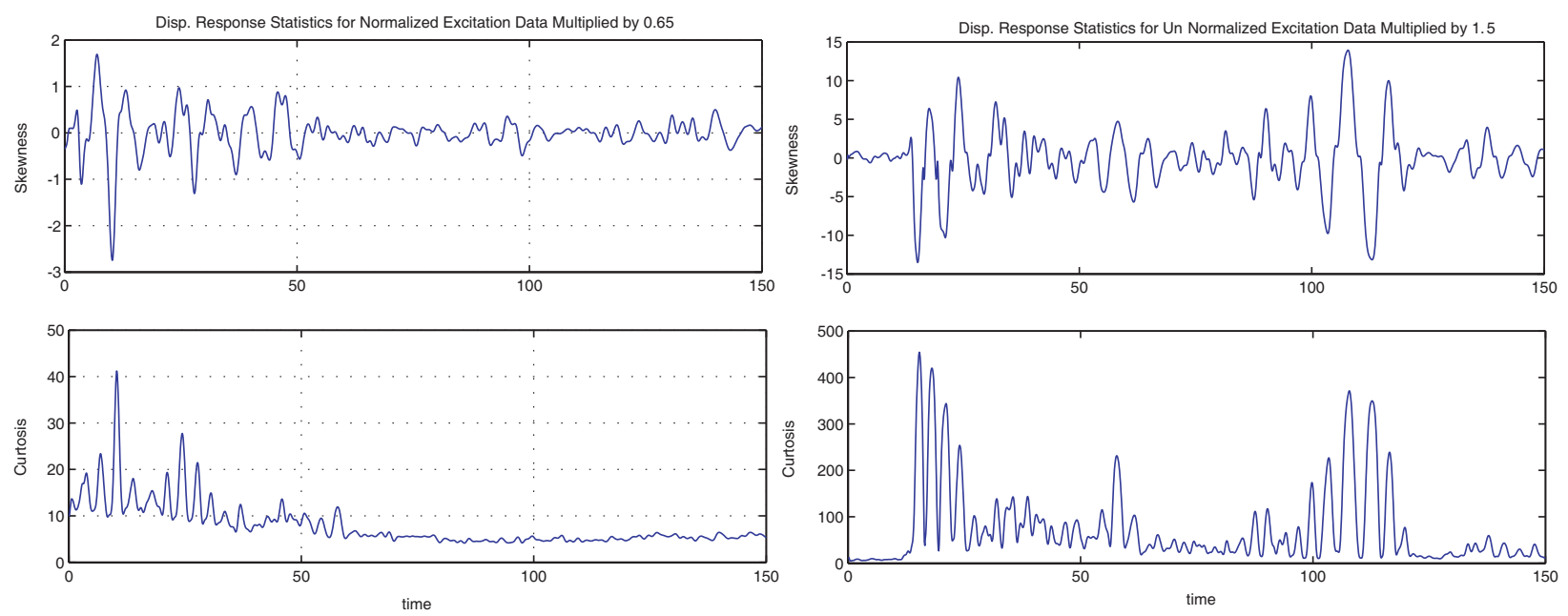

Figure 12: A comparison of the skewness and curtosis of the response displacement due to the normalized and un-normalized non-Gaussian excitation processes. Notice that the magnitude in the un-normalized case (right column) is much larger than that for the normalized case. 